\title{
Flight Muscle Development in Locusta migratoria: Effects of Implantation of Corpora Allata on the Attainment of Metabolic Enzyme Activities
}

\author{
M. A. M. Van den Hondel-Franken, A. Th. M. Van den Broek, and \\ A. M. TH. BEENAKKERS \\ Laboratory of Chemical Animal Physiology, State University of Utrecht, \\ 8 Padualaan, 3508 TB Utrecht, The Netherlands
}

Accepted January 29, 1980

\begin{abstract}
The effects of corpora allata ( $\mathrm{C} \Lambda$ ) implantation on the development of the dorsolongitudinal flight muscles and the wings and on the pigmentation in Locusta migratoria were investigated. Fifth-instar female larvae 0-24 hr after ecdysis received either one pair of CA taken from adult females or one, two, three, or four pairs of CA taken from young fourth-instar larvae. Implantation of one, two, or (except for a few cases) three pairs of larval CA did not produce any effect. However, after implantation of four pairs of larval CA, $50 \%$ of the insects arising from the subsequent molt displayed predominantly larval characteristics regarding pigmentation and wings. This phenomenon was never observed in the locusts implanted with adult CA, since the ecdysis following the implantation always resulted in the production of individuals with predominantly adult characteristics. Implantation of either adult CA or four pairs of larval CA gave rise to an extra larval stage and consequently an extra (sixth) ecdysis. During this extra larval period, flight muscle development appeared to be retarded in comparison with control insects, as was reflected by lower specific activities of enzymes indicative of aerobic metabolic pathways, such as carbohydrate oxidation and, in particular, fatty acid oxidation. Implantation of larval CA or adult CA resulted in essentially the same pattern of enzyme activities. The activity of lactate dehydrogenase, which in the control insects reached its maximum value around the fifth (imaginal) ecdysis, was in the CA-implanted locusts at its maximum value at the extra (sixth) ecdysis, which may be indicative of the period of invagination of tracheoblasts into the muscle fibers. The correlation between the JH titer and the prevention or inhibition of flight muscle developmental processes is discussed.
\end{abstract}

In the migratory locust flight muscle development takes place during the fifth larval instar and the early part of the adult stage. Brosemer et al. (1963) distinguished four phases in the developmental process: a phase of larval growth, a molting interval (characterized by a temporary cessation of growth along with the invagination of tracheoblasts into the muscle fibers), a phase of differentiation toward the final metabolic enzyme pattern in which the interfibrillar tracheoles are completed, and a phase of duplication of the enzymatic and structural muscular components. Particularly during the differentiation phase, the specific carbohydrate and fatty acid oxi- dizing properties of the flight muscles are revealed (Beenakkers et al., 1975).

Juvenile hormone $(\mathrm{JH})$, secreted by the corpora allata (CA), is involved in the regulation of larval growth and differentiation in insects (for reviews see Wigglesworth, 1970; Willis, 1974). As demonstrated by a Galleria bioassay, hemolymph JH activity in fourth-instar larvae of Locusta appeared to be at its maximum value on the first days of the instar, whereas this activity was virtually absent in the fifth larval instar (Johnson and Hill, 1973a; Joly et al., 1977). In the hemolymph of adult male and female locusts JH activity has also been found; this activity, however, has been related to the 
development of the accessory glands and ovaries (Johnson and Hill, 1973b, 1975; Joly et al., 1977). Using capillary gas chromatography with electron capture detection, only JH-III was shown to be present in both normally developing young fourth-instar female larvae and adult females (Huibregtse-Minderhoud et al., 1980).

These data concerning flight muscle morphogenesis and $\mathrm{JH}$ activity led to the suggestion that the commencement of flight muscle development is correlated with the virtual absence of $\mathrm{JH}$ in the hemolymph. Support for this hypothesis was obtained by the following experimental evidence. Allatectomy in young fourth-instar larvae induced flight muscle development already in this larval stage. The flight muscles in the premature adult arising from the subsequent molt resembled those of normally developing adult locusts including a normal mature metabolic pattern (Beenakkers and Van den Broek, 1976). On the other hand, implantation of active $\mathrm{CA}$ in young fifth-instar larvae resulted in an inhibition of flight muscle development as was demonstrated by a substantial suppression of the normal increase in protein content, particularly during the differentiation phase (Poels and Beenakkers, 1969); moreover, a change in pigmentation and a disturbance in wing development were observed (Staal, 1961; Poels and Beenakkers, 1969).

The effects of CA implantation mentioned above have been obtained with (gonadotrophic) CA taken from adult locusts (adult $C A$ ). In normal growth and development, however, prevention of flight muscle development in the larval stages preceding the fifth will be mediated by larval CA. In order to meet in fifth-instar locusts the endocrine and other physiological conditions normally present in younger larval stages, it would appear pertinent to implant CA obtained from larvae; in such experiments possible factors other than $\mathrm{JH}$ that normally could be responsible for maintaining larval characteristics in young larvae might be included also. Therefore, in our investigations, both adult $\mathrm{CA}$ and $\mathrm{CA}$ taken from fourth-instar larvae were used.

The present study deals with the influence of CA activity on the attainment of capacities of specific catabolic enzymes in the dorsolongitudinal flight muscles of the migratory locust. Furthermore, influences on pigmentation and wing development were studied as well.

\section{MATERIALS AND METHODS}

Locusts (Locusta migratoria, gregarious phase) were taken from a stock culture, reared in the laboratory at $30^{\circ}, 40 \%$ relative humidity, and a photoperiod of $12 \mathrm{hr}$. The insects were fed with reed, supplemented with rolled oats.

In the implantation experiments, fifth-instar female larvae within $24 \mathrm{hr}$ after ecdysis were used as acceptor insects. The $\mathrm{CA}$ taken from donor insects were collected in a Ringer solution $9.15 \mathrm{~g} \mathrm{NaCl}, 0.20 \mathrm{~g} \mathrm{KCl}$, $0.27 \mathrm{~g} \mathrm{CaCl}_{2} \cdot 2 \mathrm{H}_{2} \mathrm{O}, 0.61 \mathrm{~g}$ Tris, $5 \mathrm{mg}$ streptomycin sulfate; distilled water to $1000 \mathrm{ml}, \mathrm{pH} \mathrm{7.5)}$ at $0^{\circ}$, and injected with a glass needle into the acceptor insects between the first and second abdominal tergites in a volume of $25 \mu$ l of this Ringer solution. Prior to the implantation the insects were anesthetized with carbon dioxide for $1 \mathrm{~min}$. The wound was sealed with a mixture of paraffin-beeswax-colophonium $(1: 3: 3, w / w)$ to prevent loss of hemolymph.

Two series of implantation experiments were carricd out. In one series the acceptor insects received one pair of CA taken from adult females, 18 days after the imaginal ecdysis. In the other series one, two, three, or four pairs of CA taken from fourth-instar female larvae, 24-48 hr after the third larval ecdysis, were implanted. Controls were injected with $25 \mu$ l of the Ringer solution. Several days after treatment, flight muscles from a number of insects were dissected out and enzyme activities measured.

Dissected dorsolongitudinal flight muscles, freed from fat body and adhering hemolymph, were pooled and the fresh weight was determined. Unless otherwise stated the flight muscles of three to six locusts were pooled for each determination. Using an UltraTurrax disintegrator (Janke-Kunkel), the tissue was homogenized in $2-3 \mathrm{ml} 0.1 \mathrm{M}$ potassium phosphate buffer ( $\mathrm{pH} 7.3$ ) containing $2 \mathrm{mM}$ disodium EDTA and $0.074 \%$ glutathione, stirred for $30 \mathrm{~min}$, and then centrifuged at $17,000 \mathrm{~g}$ for $15 \mathrm{~min}$ (Sorvall RC-2B). The pellet was resuspended in buffer. Both the supernatant and the resuspended sediment were assayed for enzyme activities. Until determination of the enzyme activities, all manipulations were performed at $0^{\circ}$. 
Enzyme activities were measured at $25^{\circ}$, using an Eppendorf $1101 \mathrm{M}$ photometer. The activities of a number of enzymes, indicative of a certain metabolic pathway (Pette, 1965), were estimated: glyceraldehydephosphate dehydrogenase (GAPDH, EC 1.2.1.12) and lactate dehydrogenase (LDH, EC 1.1.1.27) (glycolysis), glycerophosphate dehydrogenase (GDH, EC 1.1.1.8; glycerophosphate cycle), 3-hydroxyacylCoA dehydrogenase (HOAD, FC 1.1.1.35: $\beta$ oxidation), and citrate synthase (CS, EC 4.1.3.7; citric acid cycle). The assay mixtures for these enzymes have been described by Beenakkers (1969). Protein content was determined according to Lowry et al. (1951) as modified by Schacterle and Pollack (1973).

Statistical analysis of the enzyme activities was carried out by Student's $t$ test (modified in case the variances of the means of the two samples were assumed to be unequal; Sokal and Rohlf, 1969).

\section{RESULTS}

\section{Instar Length}

Under the conditions described, the fifth larval instar period of normally developing locusts lasted about 10 days, that of the animals implanted with adult $\mathrm{CA}$ or four pairs of larval CA about 1 day less.

IImplantation of adult CA or four pairs of larval CA gave rise to an extra (sixth) molt the ecdysis of which occurred 12-30 days after the molt following implantation.

\section{Pigmentation, Wing Development, and}

\section{Flight Muscle Appearance}

With regard to the pigmentation after the ecdysis following implantation of one pair of adult CA, several categories were distinguished among the locusts, ranging from a small number of insects exhibiting typical larval coloring (such as black pigmented dorsal parts of head, pronotum, thorax, and abdomen and orange-colored ventral and lateral parts of the body) to a more extensive group of completely green individuals. All these animals were metathetelic adults with crumpled short wings. Flight muscle appearance was broad, flat, and glassy contrary to that in adult control insects where it was narrow, firm, and pink-brown colored.

Implantation of one or two pairs of larval
CA did not produce any effect, but after implantation of three pairs of larval CA in some cases a disturbed metamorphosis was observed. After ecdysis following implantation of four pairs of larval CA, about $15 \%$ of the insects displayed a normal adult pigmentation and had wings curled at the distal parts. The pigmentation of about $35 \%$ was intermediate between larval and adult and this group had crumpled wings. About 50\% of the insects, however, maintained the normal larval outer appearance such as a black pigmentation of the dorsal parts and an orange coloring of the lateral and ventral sides of the body and of the distal parts of the legs. In some cases the typical white dots on the pronotum were visible. Green pigmentation was never observed. The wings were larval, i.e., not membranous but having the function of pterotheca. Judging from the appearance, the flight muscles of these locusts closely resembled those of the insects implanted with adult $\mathrm{CA}$, whereas in the normally adult and the larval/adult pigmented locusts (as mentioned above) the flight muscles seemed to be normal, respectively less affected. We have only used the flight muscles of the "larval" group for further investigation. This group of locusts will henceforth be referred to as implanted with larval CA.

Some insects implanted with adult CA survived the extra (sixth) ecdysis resulting in an almost normal (gregarious) pigmentation and nearly normal (adult) flight muscles although somewhat less pink-brown colored and firm; only remainders of wings were present.

\section{Enzyme Activities}

The changes in the enzyme activities during the experimental period in the flight muscles of the control locusts and the insects implanted with adult CA are plotted semilogarithmically in Fig. 1 (glycolysis and glycerophosphate cycle) and Fig. 2 (TCA cycle and $\beta$-oxidation). 


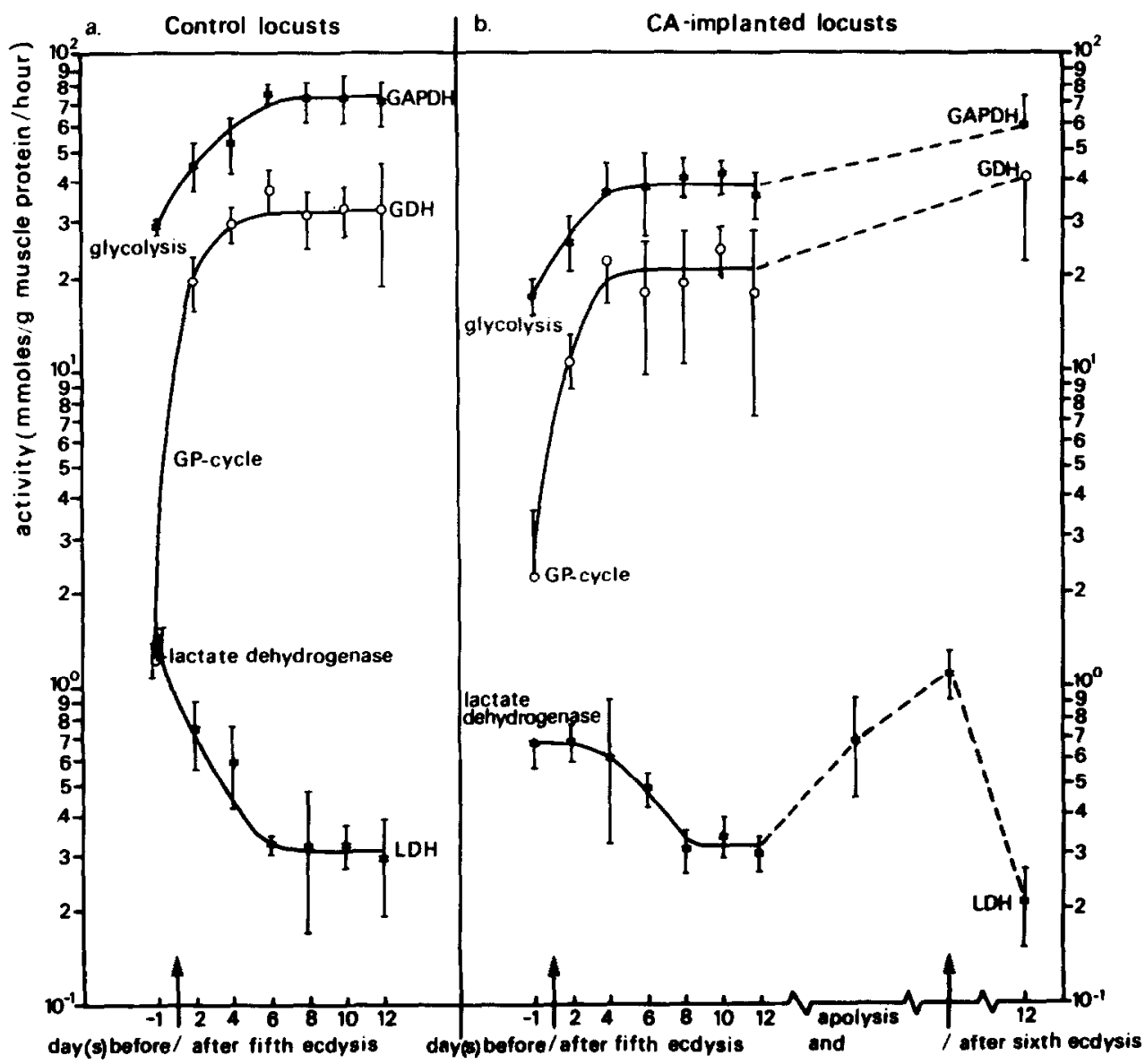

FIG. 1. Specific activities of enzymes of glycolysis and glycerophosphate cycle in the flight muscles of female locusts after: (a) injection of Ringer solution, and (b) implantation of one pair of adult CA. Each point on the solid lines represents the mean \pm SD of at least four groups of three to six insects. LDH activities at the time of apolysis and sixth ecdysis represent the mean \pm SD of 16 and 8 individuals, respectively. All enzyme activities 12 days after the sixth ecdysis represent the mean \pm SD of three individual locusts.

In the control locusts, flight muscle metabolism seems rather anaerobic on the last day before the imaginal ecdysis, judging from the relatively low CS and GDH activities and the relatively high LDH activity. The latter is obviously involved in the reoxidation of cytoplasmic NADH originating from carbohydrate degradation. During the subsequent period aerobic pathways gradually become more pronounced as reflected by the increase in CS activity together with a substantial rise in the activity of GDH, probably taking over the function of LDH that decreases to a very low level. The differentiation of the enzymes toward the final metabolic pattern takes place within the first 6 days after the imaginal ecdysis (Figs. 1 and 2).

Implantation of adult CA results in a reduction of the increase of the specific activities of GAPDH, GDH, HOAD, and CS. Although the course of the enzyme pattern has some parallel to that of the control insects, during the entire extra larval instar period the activities of these enzymes are significantly lower than the normal (adult) levels (Figs. 1 and 2).

LDH activity in the control groups was 


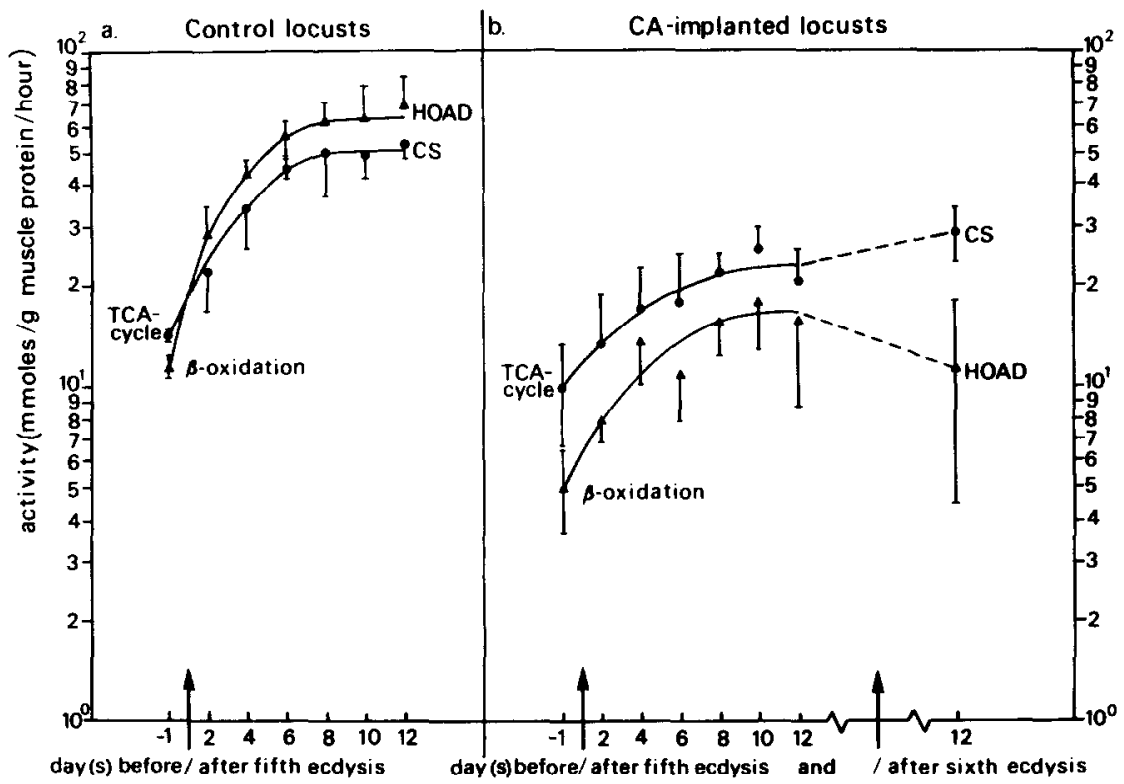

FIG. 2. Specific activities of enzymes of TCA cycle and $\beta$-oxidation in the flight muscles of female locusts after: (a) injection of Ringer solution, and (b) implantation of one pair of adult CA. Each point represents the mean $\pm \mathrm{SD}$ of at least four groups of three to six insects, except for the enzyme activities 12 days after the sixth ecdysis representing the mean $\pm \mathrm{SD}$ of three individual locusts.

highest 1 day before the fifth (imaginal) ecdysis $(1.37 \pm 0.14 \mathrm{mmol} / \mathrm{g}$ muscle protein/hr, $n=4$ ) (Fig. 1). In the groups implanted with adult CA, LDH activity was subistantially lower on the day preceding the fifth ecdysis $(0.67 \pm 0.11 \mathrm{mmol} / \mathrm{g}$ muscle protein/hr, $n=4$ ). After about 5 days activity decreased as was the case in the control groups (Fig. 1). It could be anticipated that during the subsequent molt particularly LDH activity might change again as at response to an impairment of oxygen supply. We therefore determined the LDH activity in the flight muscles of individual CA-implanted locusts on the basis of apolysis and sixth ecdysis. The highest LDH activity in the locusts implanted with adult $\mathrm{CA}$ was found at the extra (sixth) ecdysis $(1.10 \pm 0.19 \mathrm{mmol} / \mathrm{g}$ muscle protein/hr, $n=8$ ) (Fig. 1).

At the sixth ecdysis all but three locusts died. The enzyme activities in the flight muscles of the surviving locusts 12 days after the sixth ecdysis are also shown in Figi. 1 and 2. Although the data obtained are not conclusive due to the small number of insects investigated, the results suggest that the activities of the cytoplasmic enzymes (GAPDH: $58.10 \pm 16.24, \mathrm{GDH}$ : $40.24 \pm 17.99$, and LDH: $0.21 \pm 0.06$ $\mathrm{mmol} / \mathrm{g}$ muscle protein/hr) reach levels comparable to those in the control groups 12 days after the fifth ecdysis (GAPDH: $70.36 \pm 10.65(n=8), \mathrm{GDH}: 32.05 \pm 13.73$ $(n=9)$, and LDH: $0.29 \pm 0.10 \mathrm{mmol} / \mathrm{g}$ muscle protein/hr $(n=6))$. This does not hold for the mitochondrial enzymes CS and HOAD, since in the CA-implanted locusts 12 days after the sixth ecdysis the activity of CS $(28.78 \pm 5.32 \mathrm{mmol} / \mathrm{g} \mathrm{muscle}$ protein/hr) and particularly that of HOAD $(11.35 \pm 6.84 \mathrm{mmol} / \mathrm{g}$ muscle protein $/ \mathrm{hr})$ are much lower than in the control groups 12 days after the fifth ecdysis $(52.89 \pm 5.04$ ( $n$ $=7$ ), and $69.88 \pm 14.45 \mathrm{mmol} / \mathrm{g}$ muscle protein/hr $(n=7)$, respectively).

The effects of implantation of larval CA on flight muscle enzyme activities were measured on the 10th day after the fifth ecdysis. This day was considered to be ap- 
TABLE 1

Relation of Enzyme activities in the Flight Muscles of Female Locusts at the Tenth Day after the Fifth Ecdysis after (a) Injection of Ringer Solution, (b) Implantation of One Pair of adult CA, and (c) Implantation of Four Pairs of Larval CA

\begin{tabular}{lccc} 
& & \multicolumn{2}{c}{ Experiment } \\
\cline { 3 - 4 } Enzyme & (a) Ringer solution & (b) Adult CA & (c) Larval CA \\
\hline GAPDH & $73.02 \pm 12.38(5)$ & $40.69 \pm 4.35(7)^{*}$ & $44.36 \pm 9.62(5)^{*}$ \\
GDH & $32.40 \pm 5.64(5)$ & $24.21 \pm 3.82(7)^{*}$ & $19.48 \pm 6.22(5)^{*}$ \\
LDH & $0.32 \pm 0.05(5)$ & $0.34 \pm 0.05(7)$ & $0.41 \pm 0.11(5)$ \\
HOAD & $62.80 \pm 16.22(5)$ & $17.89 \pm 5.10(7)^{*}$ & $20.44 \pm 6.17(5)^{*}$ \\
CS & $48.90 \pm 7.20(5)$ & $25.30 \pm 3.96(7)^{*}$ & $27.16 \pm 5.84(5)^{*}$ \\
\hline
\end{tabular}

Note. Activities are expressed as mmol/g muscle protein $/ \mathrm{hr}$. Means $\pm \mathrm{SD}$ are given for groups of at least three locusts of which the number analyzed is shown in parentheses.

* Analysis by Student's $t$ test (see text) reveals a significant difference $(P \leqslant 0.01)$ between the control group and the CA-implanted group. Differences in enzyme activities between the two implantation experiments are not statistically significant.

propriate because the enzyme activities in the flight muscles of the control groups then have amply reached the normal adult metabolic pattern (Figs. 1 and 2). Table 1 shows these activities together with those in the control and adult CA-implanted locusts. Statistically significant differences were found between the activities of GAPDH, GDH, HOAD, and CS in the flight muscles of the control group and both CA-implanted groups. Differences between the two implantation experiments are not statistically significant (Table 1). Compared to the control values the activities of GAPDH, GDH, HOAD, and CS in the locusts implanted with CA are inhibited for around $42,33,70$, and $46 \%$, respectively.

The ratios of enzyme activities during the experimental period of the flight muscles of the control locusts and the insects implanted with adult $\mathrm{CA}$ are presented in Table 2. In the flight muscles of both control and CA-implanted locusts the relative importance of $\beta$-oxidation versus glycolysis increases during development, although in the implanted insects somewhat retarded, as is demonstrated by the decreasing GAPDH/HOAD ratio. During the entire extra larval instar, however, in the CAimplanted locusts $\beta$-oxidative capacity is most affected in comparison with other pathways, as is also shown by the HOAD/ CS ratio. The decrease of the GAPDH/CS ratio, which in the flight muscles of the control locusts takes place during the first week after the imaginal ecdysis, is delayed after CA implantation and the ratio in the implanted insects on Day 12 seems still (although not statistically significant) different from that in the control locusts. This suggests a more anaerobic nature of metabolism, even remaining in the second week after ecdysis, as is reflected by the relatively high LDH/GAPDH ratio, compared to that in the flight muscles of the control insects.

\section{DISCUSSION}

Since the virtual absence of $\mathrm{JH}$ in the fifth larval instar of Locusta will allow the subsequent ecdysis to be imaginal, it would seem likely that the most apparent conscquence of an elevation of the $\mathrm{JH}$ titer in the hemolymph of fifth-instar larvae by implantation of active CA is the inhibition of the morphological changes which normally accompany metamorphosis. In this study, the formation of new pigment patterns by the epidermal cells and the development of the wings and of the dorsal indirect flight musculature were used as parameters to investigate the effects of $\mathrm{CA}$ implantation. 

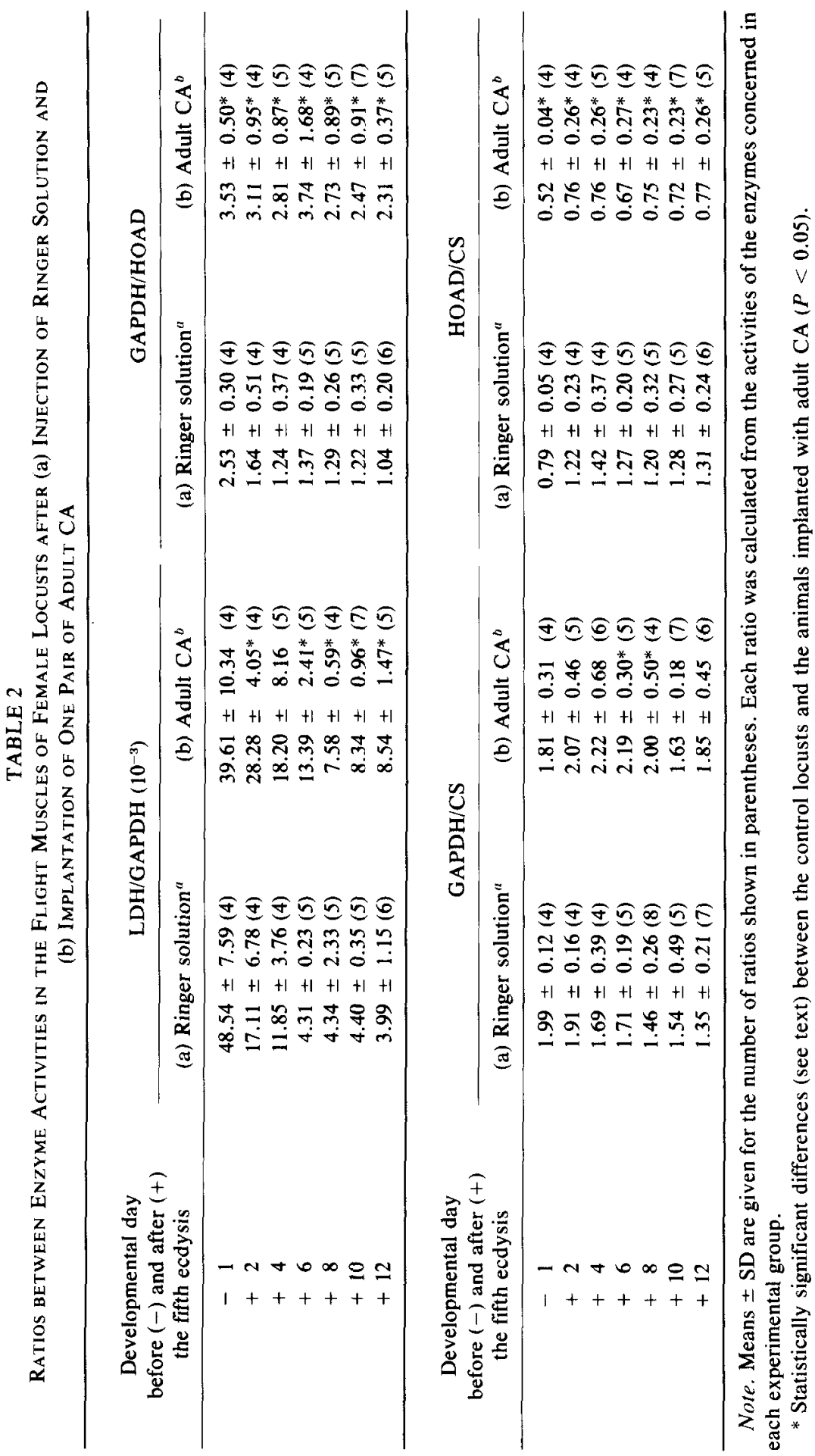
Implantation of one, two, and (except for a few cases) three pairs of larval CA did not produce any effect. Only implantation of four pairs of larval CA resulted in a disturbance in pigmentation and a deformation of the wings in most of the animals. Therefore, it may be suggested that there exists a threshold value for the $\mathrm{JH}$ titer, and maybe also for the period during which the JH titer has to be maintained on a certain level to produce any effect. On the other hand, when a comparison is made between the cffects produced after implantation of larval and adult $\mathrm{CA}$, the involvement of solely $\mathrm{JH}$ in the disturbance of pigmentation and wing development in the insects implanted with larval CA seems to be an oversimplification.

After implantation of larval CA or adult CA only $\mathrm{JH}$-III is produced, the titer being highest after implantation of adult CA; this titer remains elevated for a number of days (Huibregtse-Minderhoud et al., 1980). Despite the substantially lower JH-III titer in the locusts implanted with four pairs of larval CA, the subsequent molt could produce insects with predominantly larval characteristics. This phenomenon was never observed in the locusts implanted with adult CA. As at least a larval molt is the result of a cooperation of more hormonal factors, theoretically it is possible that an optimum $\mathrm{JH}$ titer exists for evoking a real extra larval stage. On the other hand, these results may provide evidence that a factor other than JH-III is also secreted by the larval CA which may be involved in the maintenance of the specific larval pigmentation and wings. Although JH-III itself can disturb normal pigmentation and wing development, as shown by the locusts implanted with adult $C A$, in our concept it is the balance between the level of $\mathrm{JH}$-III and the other factor which will determine to what extent typical larval characteristics will be retained.

The development of the flight muscles, however, seems to be influenced only by
JH, because compared to the control values, in the implanted insects the levels of the enzyme activities indicative of aerobic carbohydrate and fatty acid oxidation in the flight muscles were much lower independent of the type of CA used; moreover, no differences were found in the enzyme activities between the two implantation experiments. These results are fairly conclusive regarding the participation of $\mathrm{JH}-\mathrm{III}$ in the inhibition of the flight muscle development, although it cannot be determined whether this influence is direct or indirect.

From the ratios of enzyme activities in the flight muscles of the control locusts and the insects implanted with adult CA, conclusions can be drawn as to which metabolic pathway(s) is(are) most influenced by CA implantation (cf. Pette, 1965). Apparently during the whole extra larval instar period, fatty acid oxidation is predominantly suppressed. Particularly this metabolic pathway is of utmost importance in terms of energy supply for the flight muscles in the adult during prolonged flight (Beenakkers, 1969; Jutsum and Goldsworthy, 1976; Van der Horst et al., 1978).

Since CA implantation gave rise not only to an extra larval instar but consequently to an extra molt as well, the prothoracic glands apparently remained still functioning. This phenomenon, which has been demonstrated in Antheraea (Gilbert, 1962), also occurs in Locusta probably since the elevated JH-III titer prevents the degeneration of the prothoracic glands normally taking place early in the adult stage. Although during the extra molt a new cuticle had been formed, at the extra ecdysis most of the CA-implanted locusts were unable to escape from the old exuviae, possibly because of a disturbance in somatic muscle development (Sláma et al., 1974), or, more likely, because of the inability to dissociate the old wings connected to the exoskeleton from their attachments. The three locusts which survived the extra (sixth) ecdysis did possess only remainders 
of wings. The epidermis showed an almost normal adult pigmentation indicating a reversibility of the effect of $\mathrm{CA}$ implantation on this process. Though the very small number of locusts does not permit final conclusions to be drawn from the enzyme activities in the flight muscles, the results suggest that $\mathbf{1 2}$ days after the sixth ecdysis the activities of the cytoplasmic enzymes GAPDH, GDH, and LDH reach a nearly normal (adult) level. This does not hold for the mitochondrial enzymes CS and particularly HOAD, which could indicate that mitochondriogenesis is irreversibly inhibited by CA implantation. Pilot studies at the subcellular level are also suggestive of such a consequence.

Although allatectomy in young fourthinstar larvae results in the development of the flight muscles including the normal adult metabolic enzyme pattern (Beenakkers and Van den Broek, 1976), implantation of active CA in young fifth-instar larvae does not completely prevent the development of the flight muscles. This incomplete suppression would be explainable if nornal induction of flight muscle development is not only initiated by the virtual absence of $\mathrm{JH}$ in the fifth larval instar, but is depending on an overall decreasing concentration of $\mathrm{JH}$ in the preceding instars as well, as must be the case for wing development in many exopterygote insects. In this view, processes that alrcady have started, will be no longer sensitive to $\mathrm{JH}$ at the moment of CA implantation; however, particular processes taking place later in the sequence of normal development may be inhibited, for instance, the process preceding and requisite for the full development of the oxidative enzyme pattern, i.e., trachcolization. Brosemer et al. (1963) have correlated the period of invagination of tracheoblasts into the muscle fibers with that of maximum LDH activity which normally occurs around the fifth (imaginal) ecdysis. The latter aspect is confirmed by our experimental results, but in the $\mathrm{CA}$ - implanted locusts the highest LDH activity was observed at the extra (sixth) ecdysis, which may be indicative of the period of invagination of tracheoblasts into the muscle fibers.

\section{ACKNOWLEDGMENTS}

We thank Mr. E. V. M. Brouwers and Mr. J. G. van Beek for their assistance in the estimation of enzyme activities. Critical reading of the manuscript by Dr. D. J. van der Horst is gratefully acknowledged.

\section{REFERENCES}

Beenakkers, A. M. Th. (1969). Carbohydrate and fat as a fuel for insect flight. A comparative study. $J$. Insect Physiol. 15, 353-361.

Bccnakkers, A.M.Th., and Van den Broek, A. Th. M. (1976). Juvenile hormone and the development of locust muscles. Gen. Comp. Endocrinol. 29, 295.

Beenakkers, A. M. Th., Van den Broek, A. Th. M., and De Ronde, Th. J. A. (1975). Development of catabolic pathways in insect flight muscles. A comparative study. J. Insect Physiol. 21, 849-859.

Brosemer, R. W., Vogell, W., and Bücher, Th. (1963). Morphologische und enzymatische Muster bei der Entwicklung indirekter Flugmuskeln von Locusta migratoria. Biochem. Z. 338, 854-910.

Gilbert, L. I. (1962). Maintenance of the prothoracic gland by the juvenile hormone in insects. Nature (London) 193, 1205-1207.

Huibregtse-Minderhoud, L., Van den HondelFranken, M. A. M., Van der Kerk-Van Hoof, A. C., Biessels, H. W. A., Salemink, C. A., Van der Horst, D. J., and Beenakkers, A. M. Th. (1980). Quantitative determination of the juvenile hormones in the haemolymph of Locusta migratoria during normal development and after implantation of corpora allata. J. Insect Physiol., in press.

Johnson, R. A., and Hill, L. (1973a). The activity of the corpora allata in the fourth and fifth larval instars of the migratory locust. $J$. Insect Physiol. $19,1921-1932$.

Johnson, R. A., and Hill, L. (1973b). Quantitative studies on the activity of the corpora allata in adult male Locusta and Schistocerca. J. Insect Physiol. 19, 2459-2467.

Johnson, R. A., and Hill, L. (1975). Activity of the corpora allata in the adult female migratory locust. J. Insect Physiol. 21, 1517-1519.

Joly, L., Hoffmann, J., and Joly, P. (1977). Controle humoral de la différenciation phasaire chez Locusta migratoria migratoroides (R.\&F.) (Orthoptères). Acrida VI, 33-42. 
Jutsum, A. R., and Goldsworthy, G. J. (1976). Fuels for flight in Locusta. J. Insect Physiol. 22, 243-249.

Lowry, O. H., Rosebrough, N. J., Farr, A. L., and Randall, R. J. (1951). Protein measurement with the Folin phenol reagent. J. Biol. Chem. 193, 265-275.

Pette, D. (1965). Plan und Muster im zellulären Stoffwechsel. Naturwissenschaften 22, 597-616.

Poels, C. L. M., and Beenakkers, A. M. Th. (1969). The effects of corpus allatum implantation on the development of flight muscles and fat body in Locusta migratoria. Entomol. Exp. Appt. 12, 312-324.

Schacterle, G. R., and Pollack, R. L. (1973). A simplified method for the quantitative assay of small amounts of protein in biologic material. Anal. Biochem. 51, 654-655.
Sokal, R. R., and Rohlf, F. J. (1969). "Biometry. The Principles and Practice of Statistics in Biological Research." Freeman, San Francisco.

Sláma, K., Romanuk, M., and Sorm, F. (1974). "Insect Hormones and Bioanalogues." SpringerVerlag, Wien/New York.

Staal, G. B. (1961). "Studies on the Physiology of Phase Induction of Locusta migratoria migratorioides R.\&F." Ph.D. Thesis, Wageningen.

Van der Horst, D. J., Baljet, A. M. C., Beenakkers, A. M. Th., and Van Handel, E. (1978). Turnover of locust haemolymph diglycerides during flight and rest. Insect Biochem. 8, 369-373.

Wigglesworth, V. B. (1970). "Insect Hormones." Oliver \& Boyd/R. \& R. Clark Ltd., Edinburgh.

Willis, J. H. (1974). Morphogenetic action of insect hormones. Annu. Rev. Entomol. 19, 97-115. 